\title{
NOTES
}

\section{ERRORCALC ADAPTED: A BASIC microcomputer program to calculate error measures for variable block sizes}

\author{
MICHAEL M. BOLDIN and JANET L. STARKES \\ McMaster University, Hamilton, Ontario, Canada
}

The software described in this paper is a modification of the original ERRORCALC program by Pizzimenti, Weeks, and Lee (1984). The program has been modified for use with the IBM PC and PC-XT (and compatible) microcomputers.

The program is designed to calculate the five types of performance error: constant error, variable error, absolute constant error, absolute error, and total error. Constant error (CE), the measure of performance accuracy, is calculated as the mean of the algebraic (signed) error scores on each trial. Performance consistency is measured by variable error (VE), which is calculated as the standard deviation of $\mathrm{CE}$. Absolute constant error ( $|\mathrm{CE}|)$ is the absolute value of a subject's constant error. Absolute error is the mean of the unsigned performance scores. The composite error measure, total error $(\mathrm{E})$, combines both performance accuracy and consistency (where $\left.\mathrm{E}^{2}=\mathrm{CE}^{2}+\mathrm{VE}^{2}\right)$. The five error values are calculated simultaneously using the ERRORCALC program.

Input. The program allows for one of two types of data entry: either (1) the criterion goal and the raw trial performance scores for a block of trials, or (2) the previously determined trial error scores. Variable trial and block sizes up to $n=25$ (i.e., 625 trials) may be specified.

Output. Presentation of the five error measure calculations for a block of trials is provided, following the conclusion of data entry. Output is similar to that provided in the original Pizzimenti et al. (1984) program. A summary of error measure over all trial blocks for a subject is also provided. This summary may be viewed on the computer's CRT display and/or printed on a line printer.

System Requirements. This version of ERRORCALC ADAPTED (ERRORCALC-A) requires an IBM PC or PC-XT (or compatible) microcomputer. Minimum requirements are one 360K floppy disk drive, 192K RAM, and Microsoft MS-DOS 2.10 with BASIC (GW-BASIC) or IBM PC-DOS 2.10 with BASIC (BASICA). Versions of ERRORCALC have also been devised on disk operating system (DOS) for both the Radio Shack TRS-80 (Models II and III) and the Apple II microcomputers (Pizzimenti et al., 1984).

Please address correspondence to Janet L. Starkes, School of Physical Education and Athletics, McMaster University, Hamilton, Ontario L8S 4K1, Canada.
Modifications. Some modifications and enhancements were made to the ERRORCALC program in its conversion to the IBM PC format. Since the PC-XT does not have the basic language loaded into RAM from a floppy disk or hard drive. DOS must also be loaded into RAM beforehand. Thus about $192 \mathrm{~K}$ of RAM are required for effective use of the ERRORCALC-A program, since DOS requires about $40 \mathrm{~K}$ and the BASIC interpreter about $80 \mathrm{~K}$, leaving about $72 \mathrm{~K}$ for the program. Unfortunately, the BASIC workspace is limited to $64 \mathrm{~K}$; therefore, ERRORCALC-A had to be optimized to use as little of that space as possible. One such optimization is with the storage of the five error measures. In the original program, these values were stored in string variables. Typically, each string was 9 characters long, plus a 2-byte pointer and a null-byte character for each. For $n=25$ blocks, the amount of memory consumed by the strings alone was $25 \times 5 \times 11=2475$ bytes-over $2 \mathrm{~K}$. To alleviate this problem, the strings were replaced by floatingpoint variables, which require only 4 bytes. The strings' overhead was caused by adding extra spaces to each value to produce a neat, aligned output. Since floating-point variables are not stored as such, the "PRINT USING", function of BASIC was utilized. This provides aligned output for each value, using only an "image string" that instructs the computer how the output should appear. In addition, PRINT USING also rounds off each value to a specified number of decimal places (in this case 3 ). Thus another routine was removed from the program (the routine that performed the rounding off and decimal alignment).

Other modifications include easier correction of data entry errors, utilization of the 80-column (as opposed to 40-column) display width, and printer output. The printer is significantly different from that, for example, of an Apple II computer. The printer of a PC is assigned a device name (typically "LPT1:") rather than a slot number. To direct output to the printer, it must be accessed as a file (using the device name as a file name), and data must be subsequently redirected to that file instead of to the video display. The program assumes "LPT1:" to be the default device name for the printer, unless instructed otherwise.

Using DOS. To facilitate the loading of ERRORCALCA, we also devised two DOS routines. One (AUTOEXEC.BAT) is used when DOS is initially loaded into the computer and is executed automatically, and the other (EC.BAT) may be used from within DOS. These routines are simple files that contain a sequence of commands for the computer to perform. The advantage is that only one command must be typed, instead of several. Listings of these files are included with the ERRORCALC-A program. The files must be included on the diskette with 
Table 1

Files on the ERRORCALC Diskette

The following files should be placed on the diskette:

$\begin{array}{lll}\text { COMMAND.COM } & \text { DOS Command Interpreter* } \\ \text { GWBASIC } & \text {.EXE } & \text { BASIC Interpreter* } \\ \text { ERRCALC } & \text {.BAS } & \text { ERRORCALC-A Program } \\ \text { ECALCII } & \text {.BAS } & \text { ERRORCALC-A Program (Revised) } \\ \text { EC } & \text {.BAT } & \text { DOS loader routine } \\ \text { AUTOEXEC.BAT } & \text { DOS automatic loader routine } \\ \text { \{EC\}LDR } & \text {.SCR } & \text { "Loading" message file } \\ \text { \{EC\}LDR } & \text {.BAS } & \text { Title screen and loader program } \\ \text { \{EC\}END } & \text {.BAS } & \text { Notes regarding availability etc. } \\ \text { \{EC } \text { DOC } & \text {.BAS } & \text { ERRORCALC-A with documentation } \\ \text { \{EC\}DOC } & \text {.TXT } & \text { This article }\end{array}$

*These programs must be provided by the user; they are not included with the ERRORCALC program.

the program itself (ERRCALC.BAS). The diskette should be formatted for $360 \mathrm{~K}$, with the DOS system on it (use: FORMAT dr: /S, where $\mathrm{dr}$ is the drive identifier) and some form of BASIC. The diskette also should have on it the files that are listed in Table 1.
Advantages of the IBM PC. The main advantages that the IBM PC and PC-XT have to offer are: a faster microprocessor (4.77 MHz as opposed to 1 or $2 \mathrm{MHz}$ for the other computer systems), resulting in quicker calculations and faster program execution; a more powerful BASIC language and disk operating system; and greater presence in research than most other computers.

Availability. A listing and sample run of ERRORCALC is available upon request from Janet Starkes. A copy of ERRORCALC-A and the related programs may also be obtained by sending a diskette to the author. Please specify the language version requested and the type of computer system to be used.

\section{REFERENCE}

PizzimentT, M. A., WeEks, D. J., \& Lee, T. D. (1984). ERRORCALC: A BASIC microcomputer program to calculate psychophysical error measures for variable block sizes. Behavior Research Methods, Instruments, \& Computers, 16, 57-58.

(Manuscript accepted for publication May 1, 1987.) 